\title{
Structure analysis of yeast glutaredoxin Grx6 protein produced in Escherichia coli
}

\author{
Mohnad Abdalla ${ }^{1,2,3^{*}+}$ D, Wafa Ali Eltayb ${ }^{2,4^{*}+}$, Amr Ahmed El-Arabey ${ }^{2}$, Raihan Mo ${ }^{2}$, T. I. M. Dafaalla ${ }^{5}$, \\ Hamed I. Hamouda ${ }^{3}$, Eijaz Ahmed Bhat ${ }^{6}$, Annoor Awadasseid ${ }^{7}$ and Hassan Abdellha Ahmed Ali ${ }^{8}$
}

\begin{abstract}
Background: Grx6 is a yeast Golgi/endoplasmic reticulum protein involved in iron-sulfur binding that belongs to monothiol glutaredoxin-protein family. Grx6 has been biochemically characterized previously. Grx6 contains a conserved cysteine residue (Cys-136). Depending on the active-site sequences, Grxs can be classified to classic dithiol GrXs with a CXXC motif known as classes II and monothiol GrXs with a CXXS motif known as classes I, and Grx6 belongs to the class I with a CSYS motif.

Results: Our results showed how the loop between the N-terminal and C-terminal can affect the stability. When Grx6 was incubated with $\mathrm{FeSO}_{4} \cdot 7 \mathrm{H}_{2} \mathrm{O}$ and $\left(\mathrm{NH}_{4}\right)_{2} \mathrm{Fe}\left(\mathrm{SO}_{4}\right)_{2} \cdot 6 \mathrm{H}_{2} \mathrm{O}$, a disulfide bond was formed between the cysteine 136 and glutathione, and the concentration of dimer and tetramer was increased. The results presented various levels of stability of Grx6 with mutant and deleted amino acids. We also highlighted the difference between the monomer and dimer forms of the Grx6, in addition to comparison of the Fe-S cluster positions among holo forms of poplar Grx-C1, human Grx2 and Saccharomyces cerevisiae Grx6.
\end{abstract}

Conclusions: In this paper, we used a combination of spectroscopic and proteomic techniques to analyse the sequence and to determine the affected mutations and deletions in the stability of Grx6. Our results have increased the knowledge about the differences between monomer and dimer structures in cellular processes and proteins whose roles and functions depend on YCA1 in yeast.

Keywords: Grx6, Purification, Saccharomyces cerevisiae, Fe-S, Crystallization

\section{Background}

Glutaredoxin proteins (Grx) have thiol reductase activity. Glutaredoxins are necessary to reduce glutathione (GSH) as the electron donor [1]. Now, the assumptive glutaredoxin sequences consist of an active site that contains one or two conserved cysteines together with two non-conserved residues among them. Previous studies have shown that two conserved cysteines are necessary for reducing protein disulfides. In addition, only the cysteine locus at the $\mathrm{N}$-terminal is essential for the reduction of incorporated disulfides with glutathione. To date, in Saccharomyces cerevisiae, ten various glutaredoxins have

\footnotetext{
*Correspondence: mohnadabdalla200@gmail.com;

wafa.ali.113388@gmail.com; wafa@mail.ustc.edu.cn

${ }^{\dagger}$ Mohnad Abdalla and Wafa Ali Eltayb contributed equally to this work.

${ }^{1}$ Faculty of Science and Technology, Omdurman Islamic University,

Khartoum, Sudan

${ }^{2}$ School of Life Sciences, University of Science and Technology of China,

Hefei, Anhui 230027, People's Republic of China

Full list of author information is available at the end of the article
}

been found. Eight of them have been biochemically characterized, and three of five dimensions are available in the PDB database [2-4].

In addition, Grx6 regulates the glutathionylation of thiols of Golgi/endoplasmic reticulum target proteins, and as a result, it regulates the equilibrium between oxidized and reduced glutathione in the lumen of these compartments. One of the main functions of glutathionylation is to protect the cell against oxidative stress. Deletion of Grx 5 resulted in serious growth defects by inducing the accumulation of the iron in the cell. Some mutations in Grx6, but not all, have deficient phenotypes in intracellular calcium transporters, such as the Golgi Pmr1 protein. Grx6 is involved in several biological processes and found in several cellular components (Table 1). These results show that any mutation in Grx6 causes defects in the pathways of the cells. Interestingly, mutagenizing the active site Cys-136 codon of the GRX6 openreading frame (ORF) into a Ser codon makes reduction in 
Table 1 Grx6 Gene Ontology

\begin{tabular}{ll}
\hline Molecular function & 1. 2 iron, 2 sulfur cluster binding \\
2. Electron carrier activity \\
3. Glutathione-disulfide reductase activity \\
4. Iron ion binding \\
5. Metal ion binding \\
6. Protein disulfide oxidoreductase activity \\
7. Protein homodimerization activity \\
Biological process & 1. Cell redox homeostasis \\
2. Cellular oxidant detoxification \\
3. Cellular response to oxidative stress \\
4. Oxidation-reduction process \\
1. Golgi apparatus \\
2. Golgi lumen \\
3. Cis-Golgi network \\
4. Endoplasmic reticulum membrane \\
5. Fungal-type vacuole \\
6. Integral component of membrane \\
7. Vacuole
\end{tabular}

the level of $\mathrm{Ca}^{2+}$ in the ER, whereas $\mathrm{Ca}^{2+}$ accumulation occurs in the cytosol. Fiddling mutants in Grx6 show a more intense unfolded protein response compared to wild-type cells upon treatment [5].

In this work, we address a three-dimensional model of Grx6N-domain presented based on the known structure of different glutaredoxins. The Grx6N-domain shows a non-classic thioredoxin fold structure. Grx6N-domain is involved in the formation of a Grx6C domain cleft. However, Grx6N-domain sequences do not contain a cysteine residue. In addition, our studies have shown that the Grx6 cysteine locus at the C-terminal is essential for the biological activity of the Grx6 protein. Site-directed mutagenesis studies indicate that some residues in the $\mathrm{N}$-domain can affect the expression of the whole protein. Despite these observations, the specific role of $\mathrm{N}$-domain in the formation of a Grx6C domain is still not clear.

\section{Methods}

\section{Cloning, expression and purification of S. cerevisiae Grx6} in $E$. coli

To investigate the number of $\mathrm{Fe} / \mathrm{S}$ clusters in Grx6 in more detail, the coding regions of Grx6/YDL010W (without the signal peptide) were PCR-amplified from $S$. cerevisiae S288c genomic DNA and cloned into the pET22b $(+)$ vector (Novagen, Cambridge) between the NdeI and XhoI sites. Extra sequences encoding residues (LEHHHHHH) were added at the C-terminus. These plasmids were transformed into E. coli strain BL21 (Biolabs, Massachusetts) by heat shock and induced using $0.02 \mathrm{mM}$ IPTG at $16{ }^{\circ} \mathrm{C}$ for $\sim 20 \mathrm{~h}$ after the $\mathrm{OD}_{800} \mathrm{~nm}$ reached 0.8 . The cells were collected by centrifugation and resuspended in buffer $(20 \mathrm{mM} \mathrm{NaCl}, 50 \mathrm{mM}$ Tris$\mathrm{HCl}, \mathrm{pH}$ 8.0). After $20 \mathrm{~min}$ of sonication, which was followed by centrifugation at 30,000 xg for $25 \mathrm{~min}$, the supernatant was pooled and loaded onto a Ni-NTA column (GE Healthcare, Chalfont). The target protein was eluted with $250 \mathrm{mM}$ imidazole and loaded onto a Superdex 75 column preequilibrated.

\section{Site-directed mutagenesis}

The site-directed mutagenesis primer was designed (Additional file 1: Table S1), and mutations were introduced into Grx6 by PCR. The plasmid was purified using an AXYGEN ${ }^{\text {tu }}$ kit (Axygen Scientific, Beijing), and then the plasmid was cut by Dpn1 (TAKARA, Beijing) using $1 \mu \mathrm{l}$ from the enzyme, $4 \mu \mathrm{l}$ buffer $B$ and a total volume up to $50 \mu \mathrm{l}$, which was incubated overnight at $37{ }^{\circ} \mathrm{C}$. This was purified again using an AXYGE kit, and then the plasmid was linked using a CE II enzyme VAZYME (Vazyme Biotech, Nanjing) with $2 \mu$ land $4 \mu \mathrm{l}$ from the buffer and a total volume up to $20 \mu \mathrm{l}$, which was incubated in ice for $30 \mathrm{~min}$. The final reaction was transformed into competent cells. Point and deletion mutations were confirmed by DNA sequencing.

\section{Nature of the Fe-S Centre in Grx6}

The nature of the $\mathrm{Fe}-\mathrm{S}$ centrein dimer Grx6 was assessed by using a spectroscopic technique. The sample purified to a dimer contained $0.1 \mathrm{~mol}$ of Fe per mol of protein.

\section{Crystallization}

Grx6 crystals were grown at $14{ }^{\circ} \mathrm{C}$ using the sitting drop vapour diffusion method. The dimer, using $1.0 \mu$ l protein sample, was prepared as described above with an equal volume of reservoir solution (0.1 M HEPES sodium $\mathrm{pH} 7.5$ and $0.8 \mathrm{M}$ potassium sodium tartrate tetrahydrate). The monomer crystals were grown at $14{ }^{\circ} \mathrm{C}$ by the hanging drop method with the following condition: salt: $0.2 \mathrm{M}$ magnesium chloride hexahydrate; buffer: $0.1 \mathrm{M}$ tris-hydrochloride $\mathrm{pH}$ 8.5; and precipitant: $30 \%$ $w / v$ polyethylene glycol 4000.

\section{Sequence analysis, protein-ligand interaction and modelling}

The disorder profile, hydrophilicity and hydrophobicity profiles were analysed using the ExPASy [6], while protein-ligand interaction was created from the Grx6 PDB file [2], and the Grx6 $\mathrm{N}$ domain model was built by using the Phyre2 [7].

\section{Results and discussion \\ Gel filtration}

Grxs have a common active site motif $\mathrm{CxxC} / \mathrm{S}$ located at a surface loop that is accessible to GSH. Gel-filtration chromatography showed that freshly purified full lengths of Grx6 have three successive peaks: the first one has a higher oligomerization state, the second has a brown colouration characteristic of the presence of a $\mathrm{Fe}-\mathrm{S}$ 
centre as a dimer, and the third has a colourless peak as a monomer. Gel filtration analysis of freshly purified recombinant ScGrx6 (Fig. 1a) had a theoretical molecular mass of $23 \mathrm{kDa}$. The apparent molecular masses of ScGrx6 determined from the upper chromatogram at $280 \mathrm{~nm}$ were $46 \mathrm{kDa}, 92 \mathrm{kDa}$, and $184 \mathrm{kDa}$. The main peaks at the retention volumes of 50 and $70 \mathrm{ml}$ were

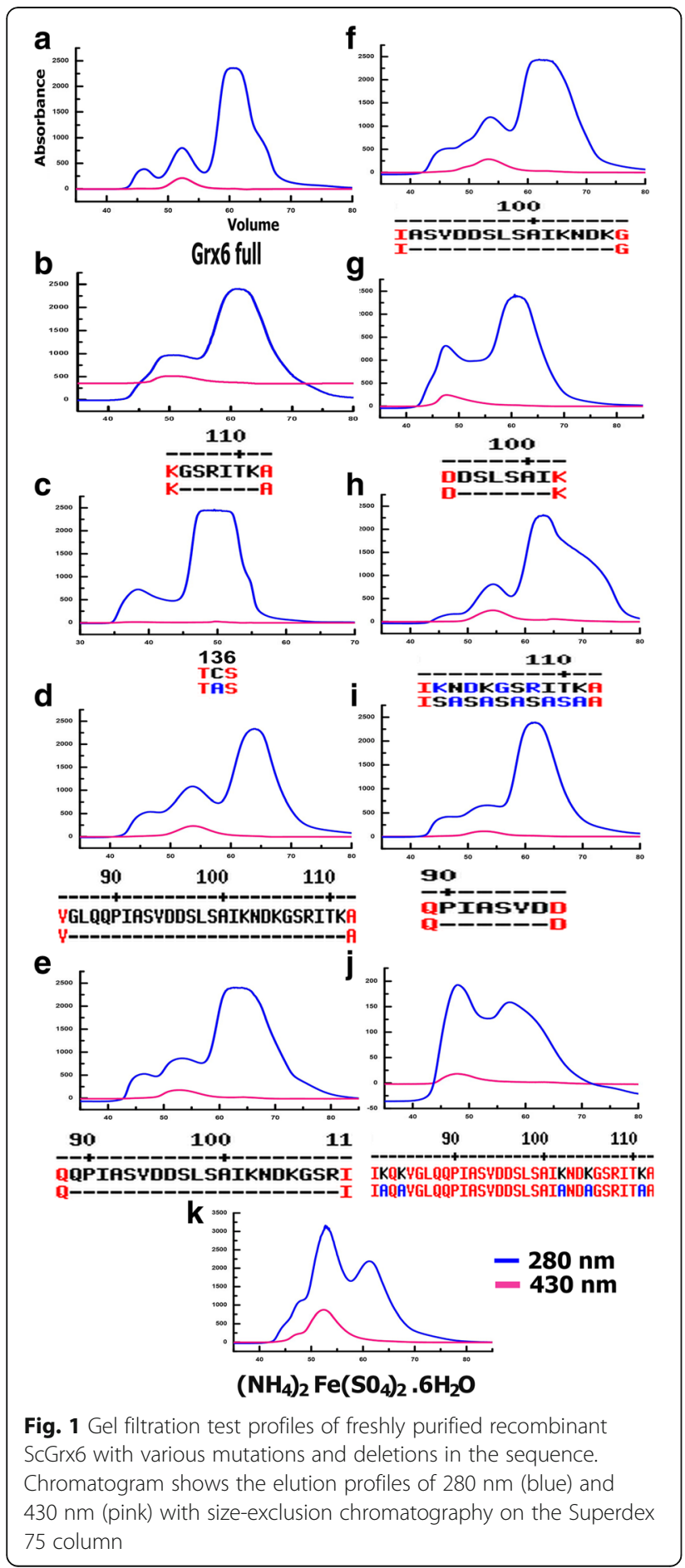

identified as oligomers (tetramer), dimer and monomers, respectively.

\section{Grx6 mutagenesis}

We created mutations and deletions in the Grx6 sequence to know the effect of them on the Grx6 purification and structure. Proteins with these mutantions and deletions were all successfully expressed as well as purified with a similar yield to the WT protein, with the exception of a mutant with five lysines replaced by alanines in positions 82, 84, 102, 105 and 111, indicating that these residues might be fundamental for the protein folding or stability. Most of the mutations and deletions showed a sharper peak pattern in the purification. We propose some mutations on the loop between the $\mathrm{N}$ and $\mathrm{C}$ domains significantly interact with the Fe-S and the GSH dependent oxidoreductase activity. To check whether the amino acids between 86 and 101, as well as the active site, are responsible for interaction with $\mathrm{Fe}-\mathrm{S}$ and the dramatically decreased GSH-dependent oxidoreductase activity of Grx6, we constructed these mutations and deletions and compared them with the wild type. The sequence requiring the assembly of a [2Fe-2S] cluster in Grxs was investigated by expressing proteins with altered active sites. Now, it is clear that the mutant $\mathrm{C} 136 \mathrm{~A}$ on the active site causes Grx6 to lose the ability to interact with Fe-S and the GSH. In fact, ScGrx6 has one cysteine (Cys) in the sequence, and this Cys is responsible for dimeric but not oligomeric conformations (Fig. 1c). UV-visible absorption studies of samples purified in the presence of GSH showed that Grx6 C136A was no longer able to incorporate a [2Fe$2 \mathrm{~S}]^{2+}$ cluster. Mutagenesis studies involving cysteine residues of Grx6 were used to investigate the cysteine residues involved in $[2 \mathrm{Fe}-2 \mathrm{~S}]^{2+}$ cluster ligation. No cluster was observed, indicating that the catalytic cysteine is likely to be a cluster ligand. The higher oligomerization state disappeared by the deletion between 96 and 101 as well as the deletion between 106 and 111 (Fig. 1b \& g). It seems that this region is important for the oligomeric state. However, it diminished when the amino acids between 102 and 111 were replaced by 5SA linkers (Fig. 1h). However, when the five lysines were replaced by alanines in positions 82, 84, 102, 105 and 111, the bacteria were not able to express Grx6. We added $\left(\mathrm{NH}_{4}\right)_{2} \mathrm{Fe}\left(\mathrm{SO}_{4}\right)_{2}$ $6 \mathrm{H}_{2} \mathrm{O}$ to increase the stability by making the protein dimer and tetramer. In addition, we found that the protein concentration increases when we add $\mathrm{FeSO}_{4} \cdot 7 \mathrm{H}_{2} \mathrm{O}$ and $\left(\mathrm{NH}_{4}\right)_{2} \mathrm{Fe}\left(\mathrm{SO}_{4}\right)_{2} \cdot 6 \mathrm{H}_{2} \mathrm{O}$ (Fig. 1k).

Our result shows how the loop between the $\mathrm{N}$-terminal and C-terminal can affect the stability and that it does not depend on the length but depends on the position of amino acid inside the loop. The loop makes significant interactions with other parts of the tetramer, indicating that the region may not be flexible in solution. 


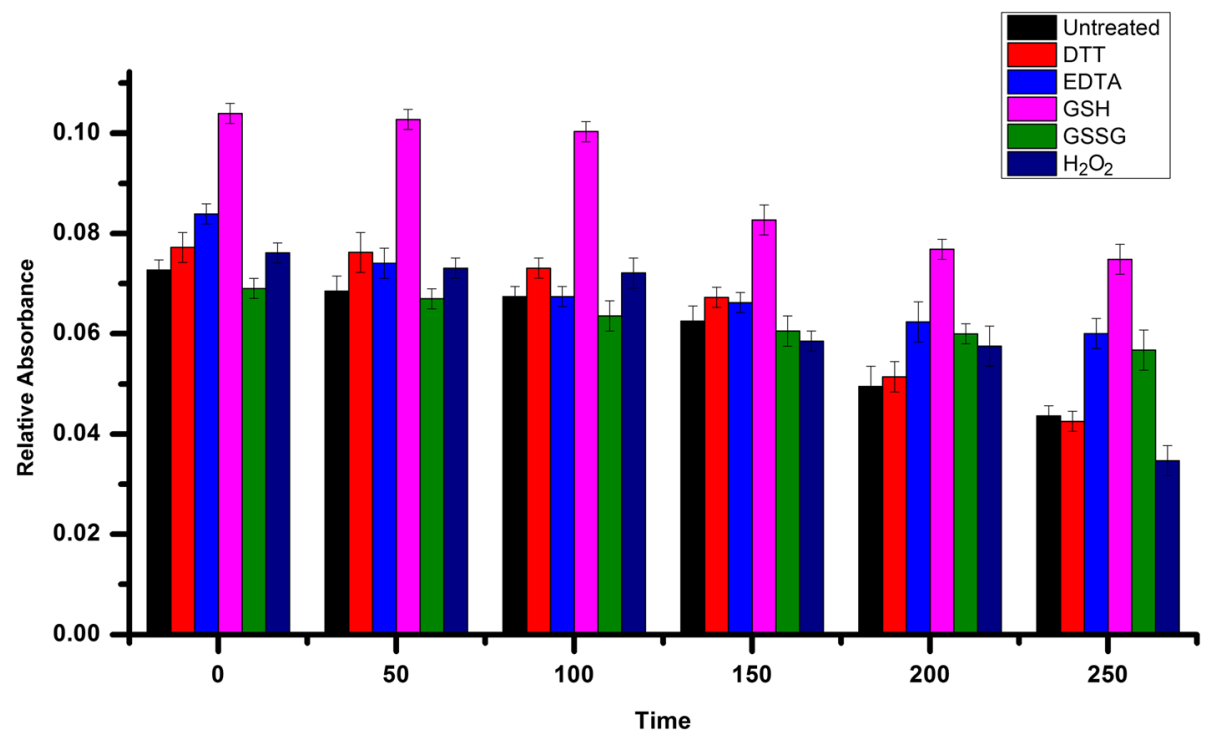

Fig. 2 ScGrx6 holoprotein stabilization in aerobic solutions by the addition of DTT, EDTA, GSH, GSSG or $\mathrm{H}_{2} \mathrm{O}_{2}$ at 2 mM. The absorbance of freshly purified ScGrx6 at $430 \mathrm{~nm}$ was measured at six different time points in the absence and presence of DTT, EDTA, GSH, GSSG or $\mathrm{H}_{2} \mathrm{O}_{2}$. The absorbance of the buffer containing $20 \mathrm{mM}$ sodium chloride and $50 \mathrm{mM}$ tris hydrochloride, $\mathrm{pH}$ 8.0, was subtracted using a reference cuvette. All assays were performed at $25^{\circ} \mathrm{C}$. The concentration of ScGrx6 in the assays was $2 \mu \mathrm{M}$. Time points: 0, 50, 100, 150, 200 and 250 min; blank is free of all additives. The experiment was repeated three times

Table 2 Comparison of Grx6 with Grxs and Grx-like domains

\begin{tabular}{|c|c|c|c|c|c|}
\hline PDB ID & Protein name & Identity (\%) & $\operatorname{RMSD}(\AA)$ & Organism & References \\
\hline 2 cq9 & GLRX2 protein & 31.73 & 3.96 & Homo sapiens & Abe, $T$ et al.(unpublished data) \\
\hline $3 d 4 m$ & Glutaredoxin-2, mitochondrial & 25.96 & 2.61 & Saccharomyces cerevisiae & [9] \\
\hline $2 \mathrm{fls}$ & Glutaredoxin-2 & 32.35 & 2.36 & Homo sapiens & Johansson et al.(unpublished data) \\
\hline $3 d 5 j$ & Glutaredoxin-2, mitochondrial & 27.18 & 2.59 & Saccharomyces cerevisiae & [9] \\
\hline $2 e 7 p$ & Glutaredoxin & 33.00 & 1.83 & Populustremuloides & [10] \\
\hline 2ht9 & Glutaredoxin-2 & 31.37 & 2.68 & Homo sapiens & [11] \\
\hline $3 c t g$ & Glutaredoxin-2 & 26.21 & 2.67 & Saccharomyces cerevisiae & [12] \\
\hline $1 z 7 r$ & Glutaredoxin & 33.33 & 2.33 & Populustremuloides & [13] \\
\hline $3 c 1 s$ & Glutaredoxin-1 & 24.04 & 2.04 & Saccharomyces cerevisiae & [14] \\
\hline 3rhe & Glutaredoxin-C5, chloroplastic & 36.36 & 2.59 & Arabidopsis thaliana & [15] \\
\hline $1 b 4 q$ & Protein (human thioltransferase) & 25.74 & 1.91 & Homo sapiens & [16] \\
\hline $3 \mathrm{~h} 8 \mathrm{q}$ & Thioredoxin reductase 3 & 31.31 & 2.74 & Homo sapiens & Chaikuad, A. et al.(unpublished data) \\
\hline 1jhb & Glutaredoxin & 23.76 & 2.21 & Homo sapiens & [17] \\
\hline 4 rqr & Glutaredoxin-1 & 23.76 & 2.55 & Homo sapiens & [18] \\
\hline 3h4k & Thioredoxin glutathione reductase & 26.32 & 2.39 & Schistosomamansoni & [19] \\
\hline $2 \times 8 g$ & Thioredoxin glutathione reductase & 26.32 & 2.50 & Schistosomamansoni & [20] \\
\hline $2 \times 99$ & Thioredoxin glutathione reductase & 26.32 & 2.50 & Schistosomamansoni & [20] \\
\hline $2 v 60$ & Thioredoxin glutathione reductase & 26.60 & 3.08 & Schistosomamansoni & [21] \\
\hline $3 d 5 j$ & Glutaredoxin-2, mitochondrial & 35.29 & 2.59 & Saccharomyces cerevisiae & [9] \\
\hline 2jac & Glutaredoxin-1 & 30.59 & 2.06 & Saccharomyces cerevisiae & [22] \\
\hline $3 \mathrm{c} 1 \mathrm{r}$ & Glutaredoxin-1 & 29.41 & 2.71 & Saccharomyces cerevisiae & [14] \\
\hline
\end{tabular}




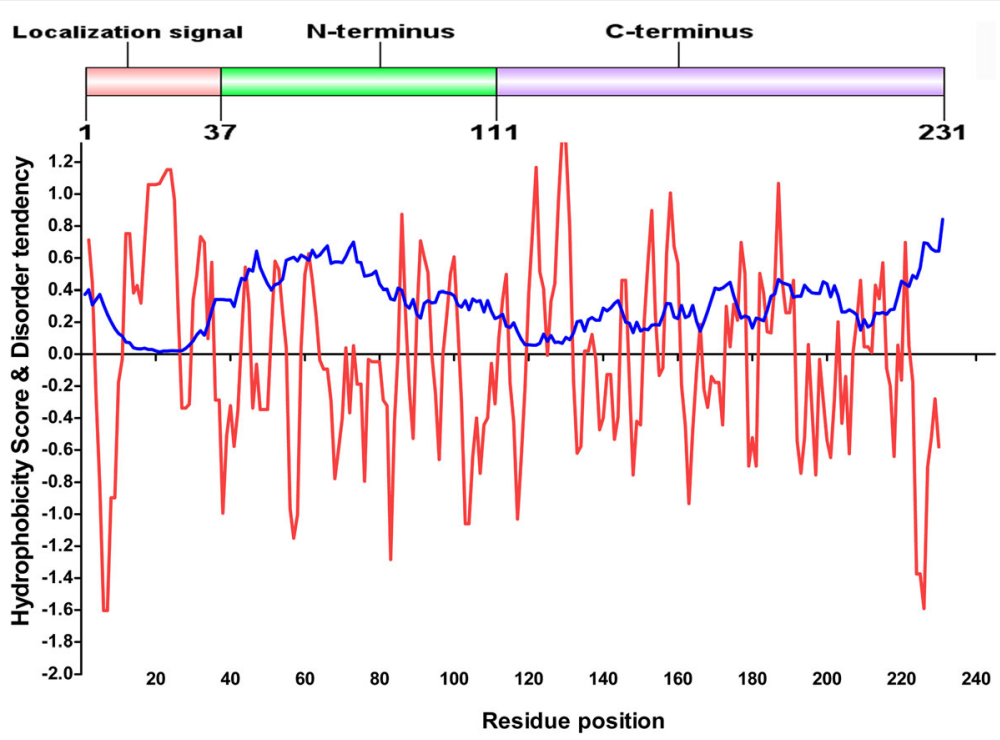

Fig. 3 A schematic representation of the ScGrx6 precursor. The regions from left to right are marked in different colours: the signal peptide 37 amino acids, N-domain 74 amino acids and C-domain 120 amino acids. The disorder profile of ScGrx6 sequences is shown underneath. Values above 0.4 are considered disordered (blue). Hydrophilicity and hydrophobicity profiles ( $>0$ indicates hydrophobicity, while $<0$ indicates hydrophilicity) are red. The numbers on the $\mathrm{X}$-axis indicate the position of the residues, and the $\mathrm{Y}$-axis indicates the relative degree of hydrophobicity and disorder. The disorder data available from http://iupred.enzim.hu/pred.php and http://web.expasy.org/protscale/

\section{Stability of Fe-S cluster}

Among the S. cerevisiae Grxs, Grx6 was the first one found to form an Fe-S cluster, and this may be a consequence of instability under aerobic conditions. The stability of the Fe-S cluster in the wild-type forms of Grx6 was observed aerobically by measuring the absorption at $430 \mathrm{~nm}$ in the presence of different oxidants and reductants as a function of time (Fig. 2). GSH, but not EDTA, GSSG or $\mathrm{H}_{2} \mathrm{O}_{2}$ stabilized the chromophore. However, the stabilizing effect was not solely restricted to the reducing capacity of GSH since ascorbic acid and dithiothreitol had a much weaker effect. In human Grx2, DTT stabilizes the cluster but to a lesser than reduced glutathione.
The cluster in adimeric form in human Grx2 was found in vivo to be inactive in Grxs classical assays [8]. However, the Grx6 dimer form has less activity than the monomer, because of the active side link to the Fe-S. The Fe-S cluster was suggested to work as a redox sensor when the Grx2 is active through conditions of oxidative stress.

\section{Sequence analysis}

The sequence identity between Grx6 and Grx-like domains is in the range $23.76-36.36 \%$. The superposition of the ScGrx6 structure with dithiol/monothiol, reduced/oxidized, GSH bound/unbound and FeS-bound/unbound forms of a

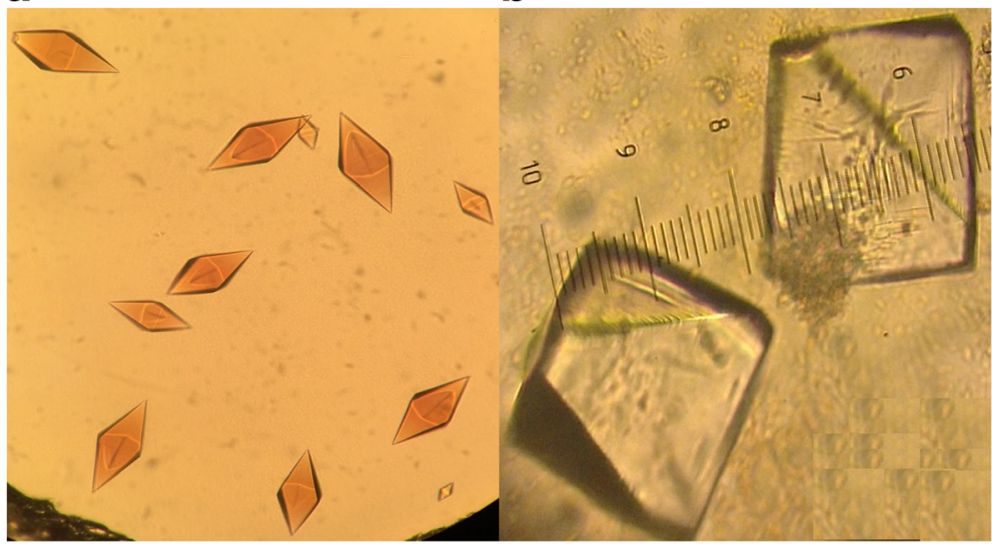

Fig. 4 ScGrx6 crystals. Dimer (a) and monomer (b) crystals 


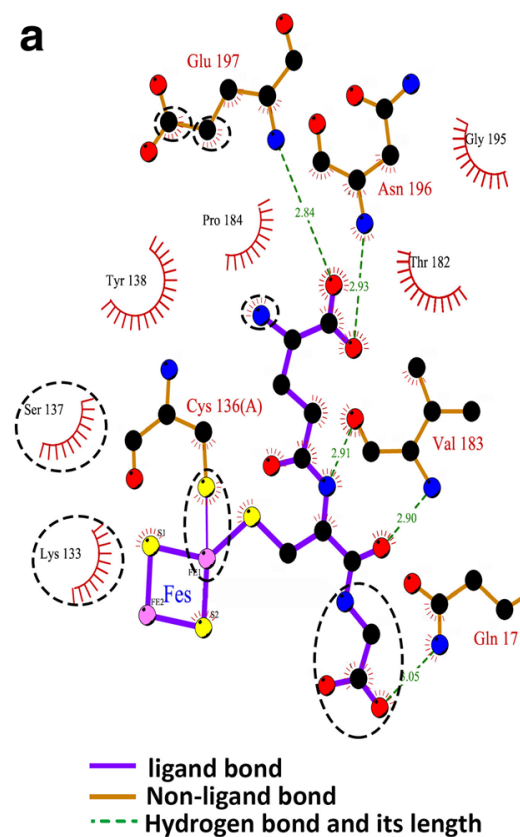

Fig. 5 Protein-ligand interaction 2D maps of dimer (a) and monomer (b); and Fe-S and GSH in the active pocket of ScGrx6. Green dashed lines between the atoms indicate hydrogen bonds with indicated distances (in $\AA$ ), whereas hydrophobic contacts are shown by an arc with spokes radiating in a direction of the ligand atoms they contact. The contacted atoms are represented with spokes encircled by back dashed lines b

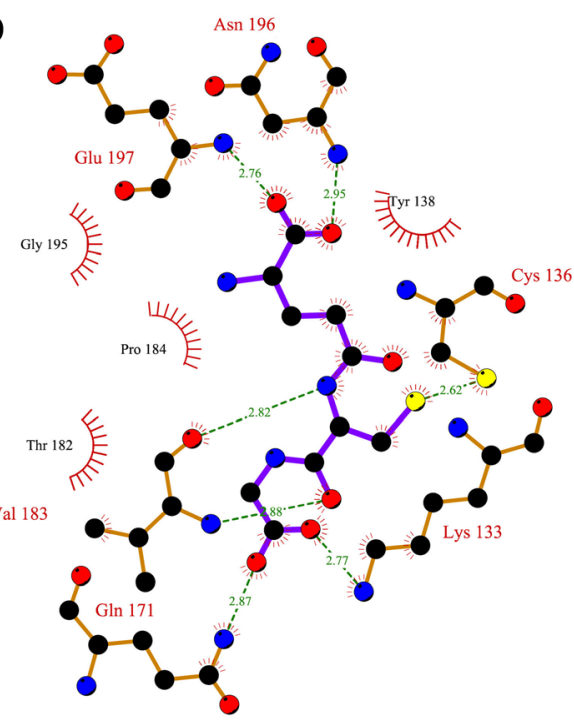

Residues involved in hydrophobic contact(s) Corresponding atoms involved in hydrophobic contact(s) OC S Fe
Grx structures from different organisms shows the root mean square deviation (RMSD) with a range of 1.83$3.96 \AA$ (Table 2), indicating that the overall folding of ScGrx6 is similar to that of other Grxs and Grx-like domains. The sequence identities and the RMSD were calculated by using the PyMOL software [23].

From these data, we can see the large disordered region from residue 42 through residue 85 . It was confirmed that the Grx6 N domain is a slightly hydrophilic peptide based on a hydrophilicity and hydrophobicity analysis. Therefore, it is a prospect that solubility of the Grx6 $\mathrm{N}$ domain will be improved with the increase of hydrophilicity of the residues (Fig. 3). It has been demonstrated in the study that the active residue $\mathrm{C} 136$ was buried in the hydrophobic region in the dimer form between the two chains of the protein (Additional file 2: Figure S1), while the higher hydrophilic residues were located at the end of the two side of the sequence. The predicted Grx6 sequence contains a cleavage site for all proteolytic enzymes shown in Additional file 3: Table S2. The Grx6 sequence contains antimicrobial domain between 131 and 142 (FSKSTCSYSKGM) Propensity 0.243 Probity $26 \%$.

\section{Grx6 crystal}

In an earlier work by Abdalla et al. [2], we determined the 3D structure of the reduced holo form of Grx6 by X-ray crystallography. Here, we have shown various differences between dimer and monomer crystals (Fig. 4). Purification chromatography indicated that Grx6 exists as a dimer and monomer in solution, while at native SDS-PAGE analysis dimer and monomer show the same molecular weight. Fe-S increases the stability of many iron-secreted proteins by protecting them against degradation and it may also be required for proper protein folding [24]. However, we realize that the Fe-S cluster may create a difficulty in protein crystallization because of oxidation. The Fe-S cluster conjugated to the secreted proteins may have intrinsic flexibility. In both cases, the dimer and monomer are suitable to protein crystallization. Thus, deletion of the C136 or the $\mathrm{C}$ domain increases the flexibility of the protein and complicates crystallization. We subjected the recombinant

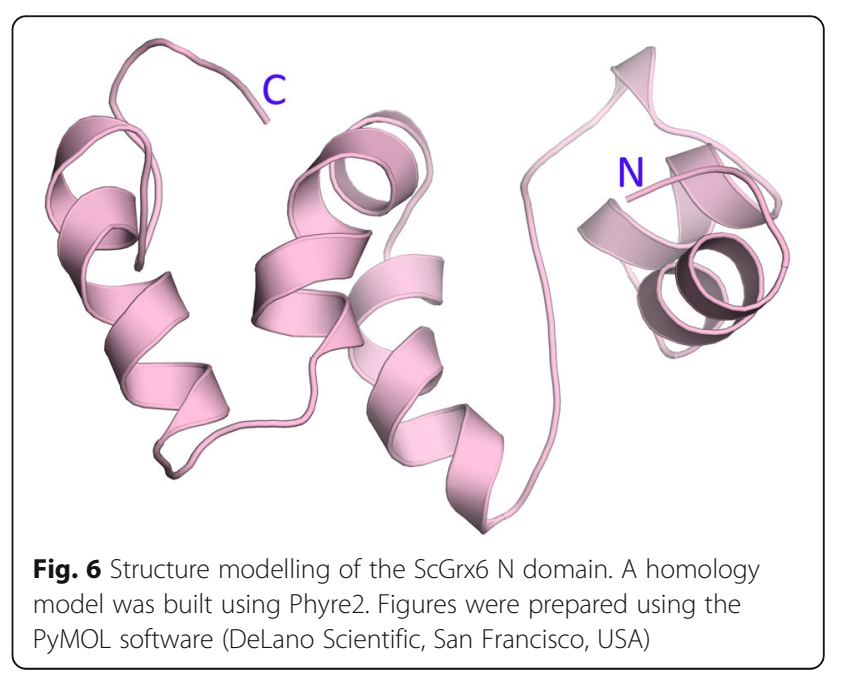




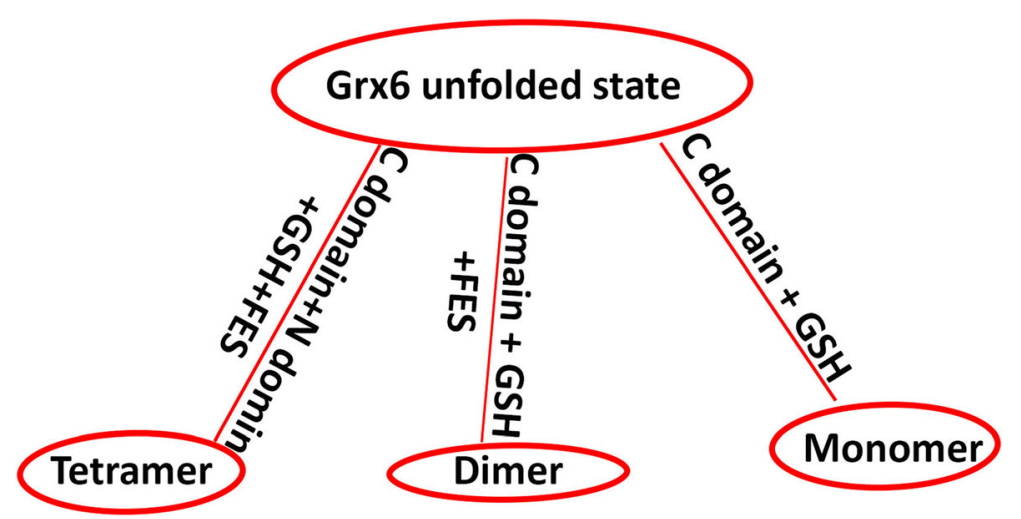

Fig. 7 Prediction of ScGrx6 structure

Grx6 protein to wide crystallization screenings. Reproducible protein crystals were obtained in $\sim 35$ conditions, and only two crystals were used in the X-ray, and they are stable, meaning that Gx6 crystals have high super saturation, viscosity and solid-liquid interfacial tension. To find full-length crystals or the N-domain crystal, we created all these mutations to circumvent this difficulty. However, all the mutation and deletion proteins are oriented to aggregate in SDS-PAGE analysis and were not suitable for protein crystallization. Both crystals diffracted to a dimer of $2.5 \AA$ and a monomer of $2.1 \AA$ resolution. Surprisingly, we obtained only the $\mathrm{C}$ domain structure. To confirm whether these crystals were indeed of full length or the $C$ domain, we isolated one crystal from both and resolved them by SDS-PAGE. Unexpectedly, there is only one band at the full length. We speculated that the $\mathrm{N}$ domain helps $\mathrm{C}$ domain to make a crystal then aggregates during the stage of $\mathrm{C}$ domain crystallization. We speculated that if we can get the $\mathrm{N}$-domain crystal, we will be able to get tetramers in the asymmetric unit of the crystal.

\section{Protein-ligand interaction}

The number of amino acid residues in the monomer and dimer involved for GSH-binding in the active pocket are the same. However, the distance of the hydrogen bonds is slightly longer in the dimer. In the monomer, there are hydrogen bonds between Cys136 and the GSH, while in the dimer, there are ligand bonds between the Cy136 and Fe-S. In addition, there are stronger hydrophobic interactions in the dimer compared to the monomer.

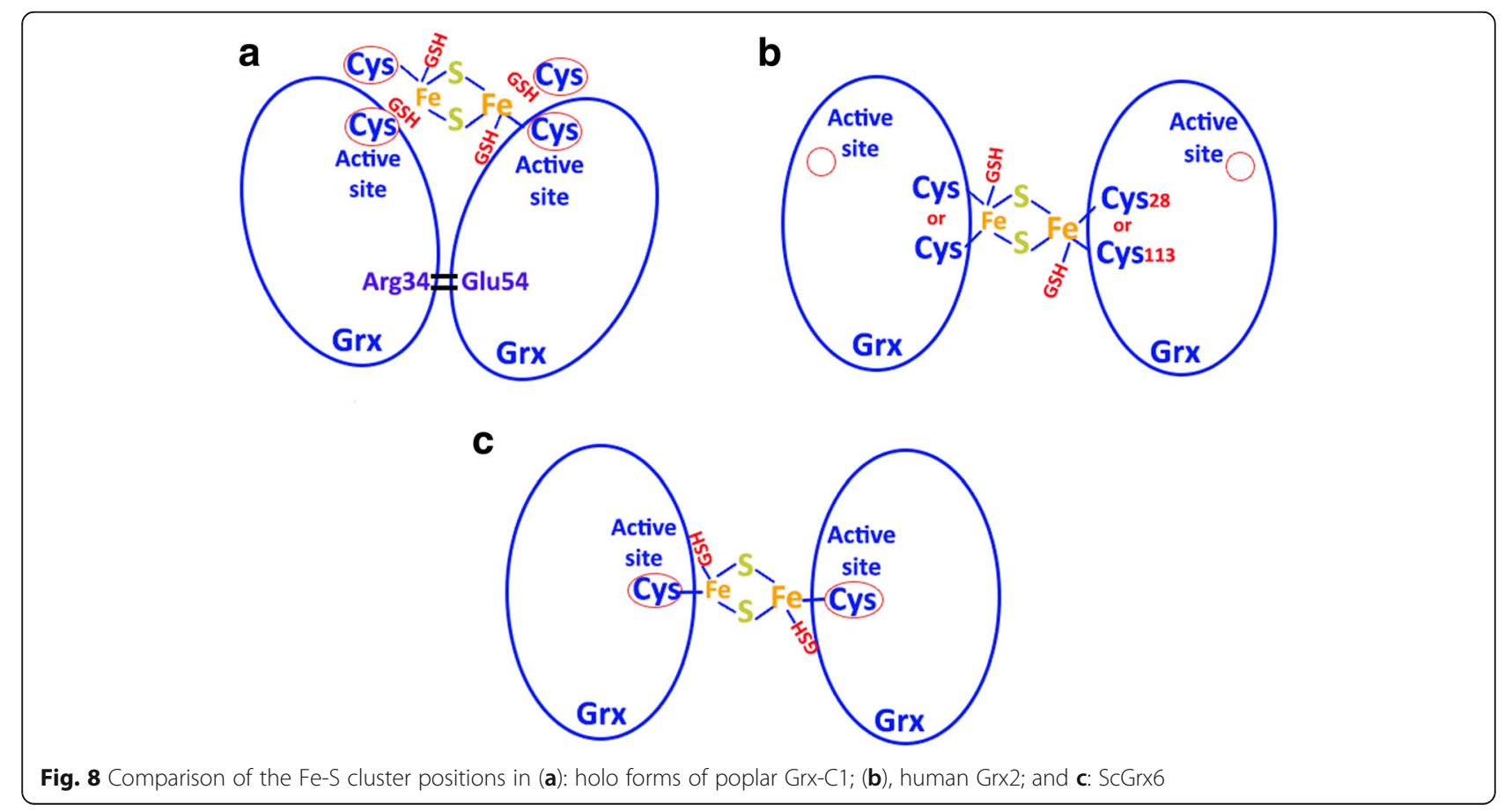


There are four amino acid residues (Tyr138, Thr182, Pro184, Gly195) involved in the hydrophobic interaction in the monomer, while there are six amino acid residues (Lys33, Ser137, Tyr138, Thr182, Pro184, Gly195). Additionally, the GSH configuration slightly changes in the dimer when compared to the monomer (Fig. 5, Additional file 4: Figure S2). Grx6 catalyses the chemical reduction of glutathione (GSH) and NADP $(+)$ which forms glutathione disulfide (GSSG), NADPH and FAD (Additional file 5: Figure S3).

\section{Modelling of the Grx6 $\mathrm{N}$ domain}

Protein sequences of Grx6 were retrieved from the Saccharomyces Genome Database [25]. The amino acid sequence alignment was performed, and BLAST was used to search for the best homology from the Grx6 N domain protein sequences. The hypothetical protein from Kazachstania africana shared the largest identity (39\%) with the target. The $\mathrm{N}$ domain sequence involves 74 amino acids, and $14.7 \%$ are Ser with a molecular weight of $8053.85 \mathrm{D}$ and a theoretical pI: 5.02. Moreover, the Grx6 N domain protein contained 12 negatively charged residues and 10 positively charged residues, and had an estimated half-life is $1.3 \mathrm{~h}$ and classifies the protein as unstable. The protein-predicted solubility upon overexpression was soluble with a probability of 0.779 . The three-dimensional structure shows that Grx6 $\mathrm{N}$ domain contains 5 alpha-helices and 6 loops (Fig. 6). The Grx6 N domain has a molecular surface area of $212.091 \AA 2$. We suppose that the $\mathrm{N}$-domain has a role in the formation of Grx6 by making it tetramer (Fig. 7).

\section{Comparison of the Fe-S cluster positions}

The iron-sulphur clusters (ISCs) in Grxs are bridging two subunits, and both of them are very unique. The ISC in holo Grx-C1 is coordinated via the active site cysteines combined with the cysteines from two GSHs, and the other two GSHs have no hydrogen bond with the Fe-S. Arg34 and Glu54 are responsible for making the tetramer in the presence of Fe-S cluster (Fig. 8a). Human Grx2 contains four cysteines that are divided into two pairs that are relatively close to each other. The first pair consists of Cys-37 and Cys-40, which are reduced and the conserved cysteine-rich motif as implicated in catalysis. The second pair consists of Cys-28 and Cys-113, which form a disulfide bond, and it is on the distal side of the molecule away from the active site cysteines (Fig. 8b). ScGrx6 is different from both of them because it has no hydrogen bonds between Arg and Glu, and it contains only one cysteine in the whole sequence (Fig. 8c).

\section{Conclusions}

In this paper, we used a combination of spectroscopic and proteomic techniques to analyse the sequence and to determine the affected mutations and deletions in the stability of ScGrx6. Our results have increased the knowledge about the differences between monomer and dimer structures in cellular processes and proteins whose roles and functions depend on YCA1 in yeast.

\section{Additional files}

\section{Additional file 1: Table S1. Site-directed mutagenesis primers. (DOCX $14 \mathrm{~kb}$ )}

Additional file 2: Figure S1. Hydrophobic interaction between the two subunit of ScGrx6. The distance between two Tyr138 residues is approximately $4.1 \AA$, which is small enough for their hydrophobic interaction. The hydrophobic interaction between hydrophobic side chains of Tyr138 from both subunits in the interface contributes to the formation of the dimeric interface and helps to stabilize the cluster by reducing the solvent accessibility. (PDF $148 \mathrm{~kb}$ )

Additional file 3: Table S2. Enzymes cleaving ScGrx6 and the positions of cleavage sites. (DOCX $16 \mathrm{~kb}$ )

Additional file 4: Figure S2. Ramachandran plot for the predicted model of dimer (A) and monomer (B) of ScGrx6. All residues are in the allowed regions. The Ramachandran plots were performed for quality assessment. Only 120 (52\%) of the total 231 residues were present for both dimer and monomer in the disallowed region, whereas no other residues were present in the generously allowed regions (Fig. 2). G-factors provide a measure of how unusual a stereochemical property is. Values below -1.0 represent high unusualness, while values below -0.5 represent the unusual property. The G-factors for main chain covalent forces and dihedral angles were calculated to be 0.42 and -0.44 , respectively for the dimer, while the G-factors for main chain covalent forces and dihedral angles were calculated to be 0.50 and -0.41 , respectively for the monomer. The overall average G-factor for the dimer was 0.19, and it was 0.28 for the monomer. The Ramachandran plot and G-factors indicate that the backbone dihedral angles, phi, and psi, in the 3D model of dimer and monomer are well within acceptable limits. The Root Mean Square Deviation (RMSD) indicates the degree to which two 3D structures are similar; the lower the value, the more similar the structures. Both template and query structures were superimposed for the calculation of RMSD (Fig. 4). The RMSD value obtained from the superimposition of dimer and monomer using PyMOL view was found to be $0.3 \AA$ over a total of 120 aligned residues. The overall quality factor, Ramachandran plot characteristics, G-factors and RMSD values confirm the quality of the dimer. (PDF 2886 kb)

Additional file 5: Figure S3. Grx6 catalyses for the chemical reduction of glutathione $(\mathrm{GSH})$ and $\operatorname{NADP}(+)$, which forms glutathione disulfide (GSSG), NADPH and FAD. The Grx6s contain cysteine in their active site making them suitable for oxidation/reduction reactions (Fig. 2). Whereas ScGrx6 can reduce ROS in the cytoplasm, ScGrx6 has the ability to reduce $\mathrm{NADP}(+)$ and ScGrx6 catalyses S-glutathionylation to protect SH-groups from oxidation and restore functionally active thiols. ScGrx6 catalyses the reaction only via a monothiol mechanism. Additionally, it has been suggested to have antioxidant capabilities, but their mechanisms are less understood. (PDF $3599 \mathrm{~kb}$ )

\section{Abbreviations}

DTT: Dithiothreitol; ER: Endoplasmic reticulum; Fe-S: Iron-sulfur; Grx: Glutaredoxin; GSH: Glutathione; GSSG: Glutathione disulfide; GST: Glutathione-S-tranferease; ISC: Iron-sulphur cluster; PDB: Protein data bank; RMSD: Root mean square deviation

Acknowledgements

We thank the Chinese Academy of Sciences for their support. 


\section{Funding}

This study was financially supported by the Chinese Academy of Sciences Scholarship to MA and WAE.

\section{Authors' contributions}

MA and WAE designed the experiments, MA carried out the experiments and analyzed the data and wrote the manuscript, WAE, AAE, RM, TIMD, HIH, $E A B, A A$ and $H A A A$ read and approved the final manuscript.

\section{Ethics approval and consent to participate}

Not applicable

\section{Consent for publication}

Not applicable

\section{Competing interests}

The authors declare that they have no competing interests.

\section{Publisher's Note}

Springer Nature remains neutral with regard to jurisdictional claims in published maps and institutional affiliations.

\section{Author details}

'Faculty of Science and Technology, Omdurman Islamic University, Khartoum, Sudan. ${ }^{2}$ School of Life Sciences, University of Science and Technology of China, Hefei, Anhui 230027, People's Republic of China. ${ }^{3}$ Qingdao Institute of Bioenergy and Bioprocess Technology, Qingdao Shi, Shandong Sheng 266000, People's Republic of China. ${ }^{4}$ Faculty of Science and Technology, Shendi University, Shendi, Nher Anile, Sudan. ${ }^{5}$ College of Education, Sinnar University, 11147 Sinnar, Sudan. ${ }^{6}$ School of Biotechnology and Graduate School of Biochemistry, Yeungnam, 280, Daehak-ro, Gyeongsan-si, Gyeongsangbuk-do 712-749, South Korea. ${ }^{7}$ Department of Biochemistry and Molecular Biology, Dalian Medical University, Dalian 116044, China. ${ }^{8}$ Faculty of Medicine, Nile Valley University, Atbara, Sudan.

Received: 14 May 2018 Accepted: 5 July 2018

Published online: 06 August 2018

\section{References}

1. Subramani J, Kundumani-Sridharan V, Hilgers RH, Owens C, Das KC. Thioredoxin uses a GSH-independent route to Deglutathionylate endothelial nitric-oxide synthase and protect against myocardial infarction. J Biol Chem. 2016;291(45): 23374-89. Available from: https://www.ncbi.nlm.nih.gov/pubmed/27587398

2. Abdalla M, Dai YN, Chi CB, Cheng W, Cao DD, Zhou K, Ali W, Chen Y, Zhou CZ Crystal structure of yeast monothiol glutaredoxin Grx6 in complex with a glutathione-coordinated [2Fe-2S] cluster. Acta Crystallogr Sect F Struct BiolCommun. 2016;72(Pt 10):732-7. Available from: https:/www.ncbi.nlm.nih.gov/ pubmed/27710937

3. Eltayb WA, Abdalla M, Samad A, EL-Arabey AA, Ghanam AR, Almahi WA. Number of cysteine interactions with the activity in GRX family. J Proteomics Bioinform. 2017;10(4):114-8. Available from: https://www.omicsonline.org/open-access/ number-of-cysteine-interactions-with-the-activity-in-grx-family-jpb-1000431. php?aid $=87704$

4. Chi CB, Tang Y, Zhang J, Dai YN, Abdalla M, Chen Y, Zhou CZ. Structural and biochemical insights into the multiple functions of yeast Grx3. J Mol Biol. 2018; 430(8):1235-48. Available from: https:/www.ncbi.nlm.nih.gov/pubmed/29524511

5. Puigpinos J, Casas C, Herrero E. Altered intracellular calcium homeostasis and endoplasmic reticulum redox state in Saccharomyces cerevisiae cells lacking Gx6 glutaredoxin. Mol Biol Cell. 2015;26(1):104-16. Available from: https:/www.ncbi.nlm. nih.gov/pubmed/25355945

6. Gasteiger E, Gattiker A, Hoogland C, Ivanyi I, Appel RD, Bairoch A. ExPASy: the proteomics server for in-depth protein knowledge and analysis. Nucleic Acids Res. 2003;31(13):3784-8. Available from: https://www.ncbi.nlm.nih.gov/pmc/articles/ PMC168970/

7. Kelley LA, Mezulis S, Yates CM, Wass MN, Sternberg MJE. The Phyre2 web portal for protein modelling, prediction and analysis. Nat Protoc. 2015;10(6):845-58. Available from: https:/www.ncbi.nlm.nih.gov/pmc/articles/PMC5298202/

8. Fidai I, Wachnowsky C, Cowan JA. Mapping cellular Fe-S cluster uptake and exchange reactions - divergent pathways for iron-sulfur cluster delivery to human ferredoxins. Metallomics. 2016;8(12):1283-93. Available from: http://pubs.rsc.org/en/ content/articlelanding/2016/mt/c6mt00193a\#!divAbstract
9. Discola KF, de Oliveira MA, Rosa Cussiol JR, Monteiro G, Barcena JA, Porras P, Padilla CA, Guimaraes BG, Netto LE. Structural aspects of the distinct biochemical properties of glutaredoxin 1 and glutaredoxin 2 from Saccharomyces cerevisiae. J Mol Biol. 2009;385(3):889-901. Available from: https://www.ncbi.nlm.nih.gov/ pubmed/18992757

10. Rouhier N, Unno H, Bandyopadhyay S, Masip L, Kim SK, Hirasawa M, Gualberto JM, Lattard V, Kusunoki M, Knaff DB, et al. Functional, structural, and spectroscopic characterization of a glutathione-ligated [2Fe-2S] cluster in poplar glutaredoxin C1. Proc Natl Acad Sci U S A. 2007;104(18):7379-84. Available from: http://www.pnas. org/content/104/18/7379

11. Johansson C, Kavanagh KL, Gileadi O, Oppermann U. Reversible sequestration of active site cysteines in a 2Fe-2S-bridged dimer provides a mechanism for glutaredoxin 2 regulation in human mitochondria. J Biol Chem. 2007;282(5):3077-82. Available from: https://www.ncbi.nlm.nih.gov/ pubmed/17121859

12. Li WF, Yu J, Ma XX, Teng YB, Luo M, Tang YJ, Zhou CZ. Structural basis for the different activities of yeast Grx1 and Grx2. Biochim Biophys Acta. 2010;1804(7): 1542-7. Available from: https://www.ncbi.nlm.nih.gov/pubmed/20417731

13. Feng $Y$, Zhong $N$, Rouhier $N$, Hase $T$, Kusunoki M, Jacquot JP, Jin C, Xia B. Structural insight into poplar glutaredoxin C1 with a bridging iron-sulfur cluster at the active site. Biochemistry. 2006;45(26):7998-8008. Available from: https://www. ncbi.nlm.nih.gov/pubmed/16800625

14. Yu J, Zhang NN, Yin PD, Cui PX, Zhou CZ. Glutathionylation-triggered conformational changes of glutaredoxin Grx1 from the yeast Saccharomyces cerevisiae. Proteins. 2008;72(3):1077-83. Available from: https://www.ncbi.nlm.nih. gov/pubmed/18473363

15. Couturier J, Stroher E, Albetel AN, Roret T, Muthuramalingam M, Tarrago L, Seidel $T$, Tsan P, Jacquot JP, Johnson MK, et al. Arabidopsis chloroplastic glutaredoxin C5 as a model to explore molecular determinants for iron-sulfur cluster binding into glutaredoxins. J Biol Chem. 2011;286(31):27515-27. Available from: https:/ www.ncbi.n/m.nih.gov/pubmed/21632542

16. Yang $Y$, Jao S, Nanduri S, Starke DW, Mieyal JJ, Qin J. Reactivity of the human thioltransferase (glutaredoxin) C7S, C25S, C78S, C82S mutant and NMR solution structure of its glutathionyl mixed disulfide intermediate reflect catalytic specificity. Biochemistry. 1998;37(49):17145-56. Available from: https://www.ncbi. nlm.nih.gov/pubmed/9860827

17. Sun C, Berardi MJ, Bushweller JH. The NMR solution structure of human glutaredoxin in the fully reduced form. J Mol Biol. 1998;280(4):687-701. Available from: https://www.ncbi.n/m.nih.gov/pubmed/9677297

18. Parker AR, Petluru PN, Nienaber VL, Badger J, Leverett BD, Jair K, Sridhar V, Logan $C$, Ayala PY, Kochat $\mathrm{H}$, et al. Cysteine specific targeting of the functionally distinct peroxiredoxin and glutaredoxin proteins by the investigational disulfide BNP7787. Molecules (Basel, Switzerland). 2015;20(3):4928-50. Available from: https://www. ncbi.nlm.nih.gov/pubmed/25793542

19. Angelucci F, Sayed AA, Williams DL, Boumis G, Brunori M, Dimastrogiovanni D, Miele AE, Pauly F, Bellelli A. Inhibition of Schistosoma mansoni thioredoxinglutathione reductase by auranofin: structural and kinetic aspects. J Biol Chem. 2009:284(42):28977-85. Available from: https://www.ncbi.nlm.nih.gov/pubmed/ 19710012

20. Angelucci F, Dimastrogiovanni D, Boumis G, Brunori M, Miele AE, Saccoccia F, Bellelli A. Mapping the catalytic cycle of Schistosoma mansoni thioredoxin glutathione reductase by X-ray crystallography. J Biol Chem. 2010;285(42):32557-67. Available from: https:/www.ncbi.nlm.nih.gov/pubmed/20659890

21. Angelucci F, Miele AE, Boumis G, Dimastrogiovanni D, Brunori M, Bellelli A. Glutathione reductase and thioredoxin reductase at the crossroad: the structure of Schistosoma mansoni thioredoxin glutathione reductase. Proteins. 2008;72(3): 936-45. Available from: https://www.ncbi.nlm.nih.gov/pubmed/18300227

22. Hakansson KO, Winther JR. Structure of glutaredoxin Grx1p C30S mutant from Yeast. Acta Crystallogr Sect D Biol Crystallogr. 2007;63(Pt 3):288-94. Available from: https://www.ncbi.nlm.nih.gov/pubmed/17327665

23. DeLano WL: Pymol: an open-source molecular graphics tool. 2002. Available from: http://www.pymol.org/

24. Abdalla M, Eltayb WA, Samad A, SHM E, Dafaalla T. Important factors influencing protein crystallization. Global Journal of Biotechnology and Biomaterial Science. 2016;2(1):025-8. Available from: https:/www.peertechz.com/BiotechnologyBiomaterial-Science/GJBBS-2-108.php

25. Cherry JM, Hong EL, Amundsen C, Balakrishnan R, Binkley G, Chan ET, Christie KR, Costanzo MC, Dwight SS, Engel SR, et al. Saccharomyces genome database: the genomics resource of budding yeast. Nucleic Acids Res. 2012:40(Database issue):D700-5. Available from: https:/www ncbinlm. nih.gov/pmc/articles/PMC3245034/ 